\title{
Electron cooling rates characterization at Fermilab's Recycler*
}

\author{
L.R. Prost ${ }^{\#}$, A. Shemyakin \\ FNAL, Batavia, IL 60510, USA
}

\section{Abstract}

A $0.1 \mathrm{~A}, 4.3 \mathrm{MeV}$ DC electron beam is routinely used to cool $8 \mathrm{GeV}$ antiprotons in Fermilab's Recycler storage ring [1]. The primary function of the electron cooler is to increase the longitudinal phase-space density of the antiprotons for storing and preparing high-density bunches for injection into the Tevatron. The longitudinal cooling rate is found to significantly depend on the transverse emittance of the antiproton beam. The paper presents the measured rates and compares them with calculations based on drag force data.

\section{INTRODUCTION}

The most important characteristic of the Recycler cooler's efficiency is the longitudinal cooling rate. This rate can be calculated in a non-magnetized model, assuming that electron beam parameters are known. On the other hand, an estimation of the rate can be made in a semi-empirical way, using the results of the drag force measurements. After explaining these approaches, we describe the cooling rate measurements and discuss the results.

\section{LONGITUDINAL COOLING RATE CALCULATIONS}

The longitudinal cooling rate in the lab frame can be expressed as a time derivative of the r.m.s. antiproton momentum width $\delta p=\gamma \sigma_{p z} M_{p}$, where $\gamma=1 / \sqrt{1-\beta^{2}}$, $\sigma_{p z}$ is the r.m.s. spread of the longitudinal velocities of antiprotons in the beam frame, and $M_{p}$ is the proton mass. Then, for a coasting antiproton beam, the longitudinal cooling rate relates to the longitudinal friction force $F_{z}$ as

$$
\frac{d}{d t}\left(\frac{\delta p^{2}}{2}\right)=\gamma M_{p} \int F_{z}\left(\vec{V}_{p}, \vec{r}_{p}\right) f_{p}\left(\vec{V}_{p}, \vec{r}_{p}\right) V_{p z} d \vec{V}_{p} d \vec{r}_{p},
$$

where $f_{p}$ is the antiprotons velocity distribution and $V_{p z}$, the longitudinal component of the antiprotons velocity in the beam frame. $\vec{V}_{p}$ and $\vec{r}_{p}$ indicate the antiprotons velocity and position dependence of the friction force and velocity distribution.

Assuming a non-magnetized model for the friction force [2] with a constant Coulomb logarithm and constant electron beam properties across the area occupied by the antiproton beam, one can express the friction force as a single integral, called Binney's formula in Ref. [3], and drop the radial dependence in Eq. (1). As shown in Ref. [4], with the additional assumptions that $f_{p}$ is Gaussian, and that the transverse velocity distribution is axially symmetrical for both electrons and antiprotons, all integrals can be carried out analytically so that Eq. (1) reduces to

$$
\begin{aligned}
& \frac{d}{d t} \delta p=-\sqrt{\frac{2}{\pi}} \frac{4 \pi m_{e} r_{e}^{2} c^{4} n_{e b} \eta L_{c} \sigma_{p z}}{\sqrt{\sigma_{e z}^{2}+\sigma_{p z}^{2}}\left(\sigma_{e x}^{2}+\sigma_{p x}^{2}\right)} \cdot f_{\text {long }}(\alpha), \\
& f_{\text {long }}(\alpha)=\frac{1-\sqrt{\frac{\alpha}{1-\alpha} \cdot \arccos (\sqrt{\alpha})}}{1-\alpha}, \alpha=\frac{\sigma_{e z}^{2}+\sigma_{p z}^{2}}{\sigma_{e x}^{2}+\sigma_{p x}^{2}} .
\end{aligned}
$$

where $\sigma_{e x}$ and $\sigma_{e z}$ are the electron transverse and longitudinal rms velocity spreads in the beam frame, $\sigma_{p x}$ is the antiproton transverse rms velocity spread in the beam frame, $m_{e}$ is the mass of the electron, $r_{e}$ is the electron classical radius, $n_{e b}$ is the electron beam density in the beam frame, $\eta$ is the fraction of the ring occupied by the electron cooler and $L_{c}$ is the Coulomb logarithm.

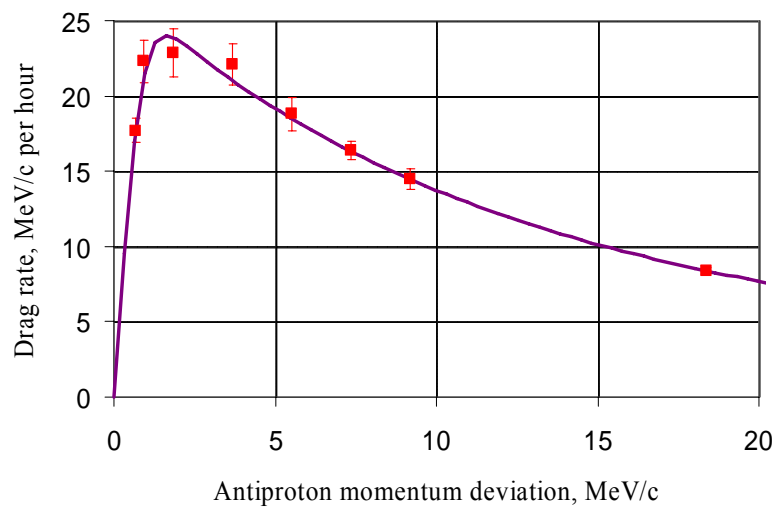

Figure 1: Drag rate as a function of the antiproton momentum deviation. $I_{b}=0.1 \mathrm{~A}$, electron beam is on axis. The solid line is a fit by Eq. (3). $N_{p}=3.5 \times 10^{10}$; the transverse emittance is $<1 \pi \mathrm{mm} \mathrm{mrad}$ (normalized, 95\%).

Eq. (2) gives the expected longitudinal cooling rate as a function of the electron beam main parameters such as its current density, its rms value of the electron angle spread and its rms energy spread. However, we found noticeable inconsistencies between independently measured electron beam parameters and those resulting from fits to drag force measurements [5]. On the other hand, if there is no correlation between the longitudinal and transverse antiproton distributions, Eq. (2) can be interpreted as a result of the integration of the function that best fits the measured drag rate data, over the antiproton distribution. Figure 1 shows the drag rates measured at various momentum deviations $p \equiv P-P_{0}=\gamma M_{p} V_{p z}$ [1] and the fitting function derived from the non-magnetized model in a form optimized for the fitting algorithm [4]: 


$$
F_{l z}(p)=-F_{0} \cdot \int_{0}^{p / p_{1}} \frac{e^{-u^{2}} u^{2}}{u^{2}+\left(\frac{p}{p_{2}}\right)^{2}} d u,
$$

where the fitting parameters $p_{1}, p_{2}$ and $F_{0}$ relate to the electron beam parameters as follows:

$$
\begin{aligned}
& p_{1}=\sqrt{2} \gamma M_{p} \sigma_{e z}, \\
& p_{2}=\sqrt{2} \gamma M_{p} \sqrt{\sigma_{e x}^{2}+\sigma_{p x}^{2}-\sigma_{e z}^{2}}, \\
& F_{0}=\frac{\gamma n_{e b}}{\sigma_{e x}^{2}+\sigma_{p x}^{2}-\sigma_{e z}^{2}} \frac{2}{\sqrt{\pi}} \frac{4 \pi m_{e} r_{e}^{2} c^{2} \eta L_{c}}{\gamma^{3} \beta^{2}} .
\end{aligned}
$$

Feeding these parameters into Eq. (2) gives a prediction of the cooling rate for the case where the antiproton beam emittance is as small as it was during the drag rate measurements. For the fit shown in Figure 1 and $\delta p=3.7 \mathrm{MeV} / \mathrm{c}$, Eq. (2) gives an expected cooling rate of $16 \mathrm{MeV} / \mathrm{c}$ per hour.

In operation, the transverse emittance of the antiproton beam is $5-10$ times larger than it is during drag rate measurements. Still, Eq. (2) predicts only a slight decrease of the cooling rate because the drag force is determined primarily by electron transverse velocities. For instance, in the range of $1<\delta p<4 \mathrm{MeV} / \mathrm{c}$ and $\varepsilon_{n}=6 \beta_{f} \sigma_{p x}^{2} / \beta \gamma c^{2}<6 \pi \mathrm{mm} \cdot \mathrm{mrad}\left(\beta_{f} \approx 30 \mathrm{~m}\right.$ is the average beta function), the calculated cooling rate given by Eq. (2) varies only by $\pm 10 \%$.

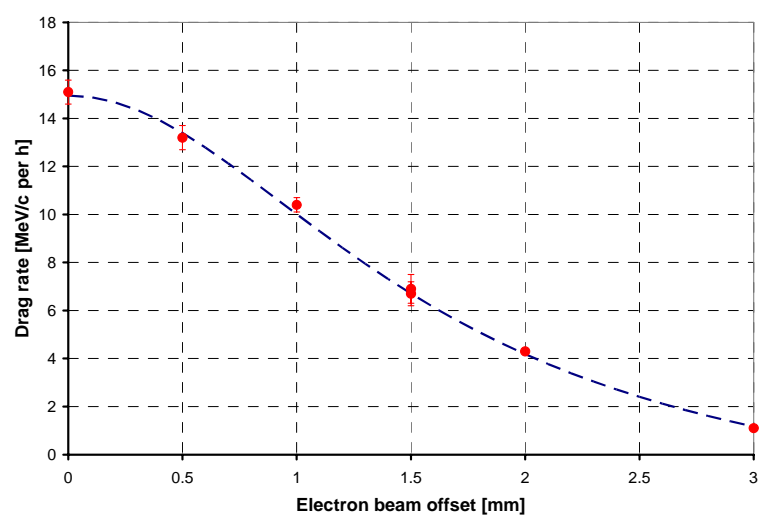

Figure 2: Drag rate as a function of the electron beam offset w.r.t. the antiprotons central orbit, for $p \equiv 3.7 \mathrm{MeV} / \mathrm{c} . I_{b}=100 \mathrm{~mA}$. The dotted blue line is the fit to the data using Eq. (6) $(a=3.72, b=1.61)$. The difference with the maxima found in Figure 1 is attributed to the deterioration of the electron beam quality between the two data sets (taken several months apart).

However, for large emittances, antiprotons spend a significant amount of time outside of the electron beam, which makes the model inapplicable. In addition, the drag force falls off rapidly with the offset of the electron beam with respect to the antiprotons central orbit (Figure 2). At a typical emittance $\varepsilon_{n} \approx 8 \pi \mathrm{mm} \operatorname{mrad}(95 \%$, normalized), the rms beam radius is $2 \mathrm{~mm}$, so that a significant portion of the beam is only weakly affected by electron cooling. Therefore, in the interesting range of parameters, we can ignore the weak dependence of the cooling dynamics on the antiproton transverse velocity and only take into account the radial decrease of the drag force. Furthermore, the strongest contribution to the radial dependence is expected to come from $F_{0} \propto n_{e b} / \sigma_{e x}^{2}$, which does not depend on the antiproton longitudinal velocity. Hence, the drag force across the beam can be interpolated as

$$
F_{z}\left(\vec{V}_{p}, r\right) \approx F_{l z}(p)_{\sigma_{p x}=0} \cdot F_{r}(r) .
$$

We then assume that the radial dependence normalized by its maximum, $F_{r}(r)$, stays the same for all momenta and can be expressed as

$$
F_{r}(r)=\left(1-\left(\frac{r}{a}\right)^{2}\right) \cdot\left(1+\left(\frac{r}{b}\right)^{2}\right)^{-1},
$$

where $a$ and $b$ are fitting parameters $\left(F_{r}(r)=0\right.$ for $\left.r>a\right)$.

In this approximation, for a Gaussian current density distribution of the antiprotons, the integral of Eq. (1) reduces to a product of its momentum and radial parts:

$$
\begin{aligned}
& \frac{d}{d t} \delta p \approx G(p) \frac{1}{\sigma_{x}^{2}} \int_{0}^{\infty} e^{-\frac{r^{2}}{2 \sigma_{x}^{2}}} F_{r}(r) \cdot r d r \\
& G(p)=\left(\frac{d}{d t} \delta p\right)_{\sigma_{p x}=0},
\end{aligned}
$$

where $\sigma_{x}$ is the rms size of the antiproton beam. Thus, Eq. (7), which can be computed numerically (see Fig.4), gives the longitudinal cooling rate as a function of the antiproton emittance.

\section{COOLING RATE MEASUREMENTS}

Cooling rates are measured according to the following procedure. Initially, the antiproton beam, confined by rectangular RF barriers, is cooled with the stochastic cooling system only in order to achieve antiproton momentum and transverse distributions as close as possible to Gaussian. Before the measurement starts, the bunch length is adjusted such that $\delta p \sim 3.5 \mathrm{MeV} / \mathrm{c}$. The measurement consists of two parts. First, the stochastic cooling system is turned off and the antiproton beam is let diffuse for 15 minutes. Then, the electron beam is turned on, and cooling is carried on for another 15 minutes. During the measurement, $\delta p$, the transverse emittances measured with a Schottky detector, $\varepsilon_{S c h}$, and transverse emittances obtained from flying wire measurements, $\varepsilon_{F W}$, are recorded.

The longitudinal/transverse cooling rates reported below are calculated as the difference between time derivatives of the momentum spread/emittances before and after turning on electron cooling. In the example of the measurement shown in Figure 3, the longitudinal cooling rate is found to be $-7.3 \pm 0.4 \mathrm{MeV} / \mathrm{c}$ per hour; the average transverse cooling rate $-5.5 \pm 0.3 \pi \mathrm{mm} \mathrm{mrad} / \mathrm{hr}$ 
for the flying wire data and $-2.4 \pm 0.1 \pi \mathrm{mm} \mathrm{mrad} / \mathrm{hr}$ for the Schottky detector data.

Longitudinal cooling rates measured for various values of the transverse emittance of the antiprotons are shown in Figure 4.

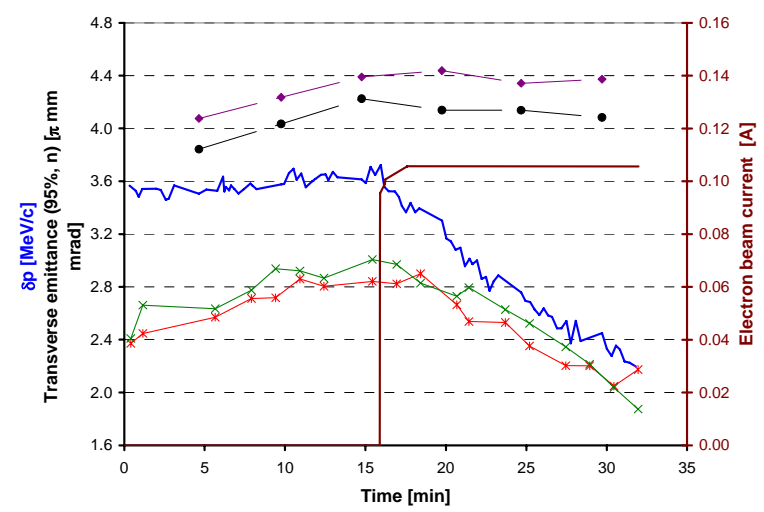

Figure 3: Evolution of $\delta p$ (blue line) and of the transverse emittances (normalized, 95\%) during a cooling rate measurement sequence. Two sets of transverse emittances are plotted: from Schottky detector data (red star: horizontal, green cross: vertical) and from flying wire measurements (purple diamond: horizontal, black circle: vertical). The brown line indicates when the electron beam was turned on and set to $100 \mathrm{~mA} . N_{p}=36 \times 10^{10}$, bunch length $=5.4 \mu$ s.

The solid curve is a fit using Eq. (7) where the only fitting parameter, $G(3.7 \mathrm{MeV} / \mathrm{c})$, is found to be $-12.5 \mathrm{MeV} / \mathrm{c}$ per hour, in reasonable agreement with the value obtained from Eq. (2) and (3), -16 MeV/c per hour, which solely relies on on-axis drag rate measurements.

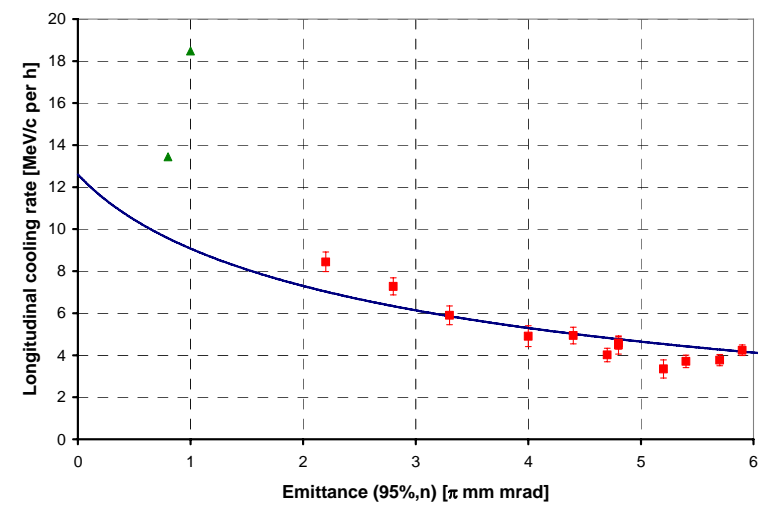

Figure 4: Longitudinal cooling rate (negated) as a function of the antiprotons transverse emittance measured with flying wires. The blue line is a fit of the data (red squares) using Eq. (7) fed with Eq. (6). Error bars are the standard error value from fitting the cooling rate data to linear slopes. The data points for $\varepsilon_{\mathrm{FW}} \leq 1 \pi \mathrm{mm} \mathrm{mrad}$ (green triangle) were obtained for a coasting beam with a low number of particles. The electron beam was on axis for all measurements.
The two data points for which $\varepsilon_{F W} \leq 1 \pi \mathrm{mm}$ mrad were acquired for beam conditions standard in drag rate measurements (coasting beam, $\delta p<1 \mathrm{MeV} / \mathrm{c}$ ). Eq. (2) was used to correct for the rms momentum spread difference with the rest of the data plotted on Figure 4 where $\delta p \approx 3.5-3.7 \mathrm{MeV} / \mathrm{c}$. The faster cooling rates measured for these cases are currently not understood.

The measurements were made for a number of antiprotons $N_{p}=30-200 \times 10^{10}$ particles to avoid the use of electron cooling at the preparation stage. Even a short time with the electron beam on axis changes the antiproton transverse distribution noticeably. It was found that the ratio $R e=\varepsilon_{S c h} / \varepsilon_{F W}$ can be used as a measure of the deviation of the distribution from Gaussian, because the Schottky emittance is sensitive to the tail population but the flying wire data is fitted to a Gaussian curve, effectively truncating the tails of the acquired distribution. While there is a significant unexplained discrepancy in the absolute numbers reported by these two diagnostics, their ratio is stable for near-equilibrium, stochasticallycooled distributions, $R e=1.5-1.7$. For a deeply electroncooled beam, this ratio can be as high as 5, and for the 15-min cooling measurements shown above, it takes 0.5 - 1 hour for $R e$ to relax back to the stochasticallycooled value. As a result, typically only one such measurement was made between injections into the Recycler ( $2 \mathrm{~h})$.

Interpretation of the transverse emittance measurements is more difficult and their detailed analysis is still in progress.

\section{CONCLUSION}

The longitudinal electron-cooling rates are measured with an antiproton beam distribution close to Gaussian. The results are found to be in reasonable agreement with predictions using a semi-empirical model based on the measured dependence of the drag force on the momentum deviation and the radial offset. The longitudinal cooling rate significantly depends on the antiprotons transverse emittance, decreasing from its peak value by a factor of $\sim 3$ for emittances typical at injection, $\varepsilon_{F W} \sim 6 \pi \mathrm{mm} \mathrm{mrad}$ (95\%, normalized).

\section{ACKNOWLEDGMENTS}

The authors are grateful to A. Burov and V. Lebedev for fructuous discussions regarding the theoretical aspects of the paper. We also would like to thank M. Hu and D. Broemmelsiek for their efforts with the beam diagnostics. Discussions with A. Fedotov and A. Sidorin and their participation in a set of measurements are appreciated.

\section{REFERENCES}

[1] A. Shemyakin et al., Proc. of EPAC'06, Edinburgh, UK, June 26-30, 2006, TUPLS069, 1654-1656

[2] Ya.S. Derbenev and A.N. Skrinsky, Particle Accelerators 8 (1977) 1 
[3] I.N. Meshkov et al., Physics guide of BETACOOL code, v.1.1, BNL note C-A/AP\#262, p. 17

[4] A. Shemyakin, FERMILAB-TM-2374-AD

[5] L. Prost et al., in Proc. of HB2006, Tsukuba, Japan, May 29-June 2, 2006, WEAY02, p 182 